\title{
ESTIMATIVA DE PARÂMETROS CINÉTICOS DA PIRÓLISE DO BAGAÇO DE LARANJA
}

\author{
T. S. LIRA ${ }^{1}$, T. P. XAVIER ${ }^{1}$ e L. C. BENEVIDES ${ }^{1}$ \\ ${ }^{1}$ Universidade Federal do Espírito Santo, Departamento de Engenharias e Tecnologia \\ E-mail para contato: taisa.lira@ufes.br
}

\begin{abstract}
RESUMO - Na busca de alternativas para amenizar os problemas ambientais causados pelo uso de combustíveis fósseis, tem-se estudado o desenvolvimento de tecnologias "limpas" e renováveis. Dentre essas tecnologias, está a obtenção de energia a partir da pirólise de biomassa. A compreensão do processo de pirólise envolve o estudo da cinética de degradação da biomassa, dos fenômenos de transporte, bem como do tipo, configuração e condições ótimas de operação do reator. O Brasil é o maior produtor de laranjas no mundo e utiliza mais de $70 \%$ para fabricação de seu suco, gerando grande quantidade de resíduo (bagaço). Dessa forma, este trabalho propôs estimar os parâmetros cinéticos das reações de pirólise de bagaço de laranja. Para isto, análises termogravimétricas dinâmicas foram realizadas e as energias de ativação foram obtidas a partir dos modelos de Ozawa, K-A-S e Starink.
\end{abstract}

\section{INTRODUÇÃO}

Devido ao constante aumento da demanda por fontes de energia e a necessidade de redução da poluição ambiental, têm-se incentivado pesquisas no sentido de se desenvolver novas fontes de energia limpa e renovável para diversas áreas industriais, visando reduzir ou substituir os combustíveis de origem petrolífera (Gamba, 2009). Neste sentido, surge a obtenção de energia a partir da pirólise de biomassa, mais especificamente a pirólise do bagaço de laranja.

A citricultura brasileira está altamente concentrada na produção de laranjas doces, com cerca de $90 \%$ do total da produção nacional de citros, sendo a região sudeste a que mais se destaca, com aproximadamente $82 \%$ da produção nacional (IBGE, 2014). O Brasil detém $50 \%$ da produção mundial de suco de laranja, sendo esta a bebida à base de frutas mais consumida no mundo, com $35 \%$ de participação entre os sucos. Isso ocorre devido a seu elevado valor nutricional, alto índice de fibras, água e vitaminas; o consumo de uma unidade dessa fruta corresponde à quantidade recomendada de dose diária de vitamina $\mathrm{C}(60 \mathrm{mg})$, o que equivaleria ao consumo de 15 maçã s (CitrusBR, 2014).

O ranking de maior país produtor de suco de laranja faz com que o Brasil gere uma grande quantidade de resíduos desta fruta, o bagaço de laranja, que correspondem a 50\% do peso da fruta (Corazza et al., 2001). Assim, a crescente preocupação com a quantidade de resíduos sólidos produzidos, reunidos ao desenvolvimento de tecnologia, viabilizam o aproveitamento e reciclagem cada vez maior dos resíduos, promovendo economia de recursos naturais, geração de empregos diretos e indiretos e redução do volume de material a ser disposto (Silveira, 2008). Deste modo, a produção de energia utilizando-se de bagaço de laranja tem sido uma alternativa viável. 
O aproveitamento de biomassa para produção de energia pode ser feito utilizando-se da combustão em fornos e caldeiras, sendo esta uma das principais vantagens da biomassa. Com objetivo de aumentar-se a eficiência do processo tem-se desenvolvido e aprimorado tecnologias de conversão mais eficientes, como a pirólise e a gaseificação (ANEEL, 2005).

A pirólise é o mais antigo e o mais simples processo de conversão de um combustível em outro de melhor conteúdo energético. Este processo possui reações químicas complexas que estão combinadas com processos de transferência de massa e calor, consistindo no aquecimento da biomassa em atmosfera não oxidante, normalmente entre $300^{\circ} \mathrm{C}$ e $500^{\circ} \mathrm{C}$, dando lugar à formação de um resíduo sólido rico em carbono (carvão) e uma fração volátil composta de vapores orgânicos e gases condensáveis (licor pirolenhoso) (ANEEL, 2005). A pirólise pode também ser empregada no aproveitamento de resíduos vegetais, como subprodutos de processos agroind ustriais. Nesse caso, os resíduos são compactados, sendo transformados em briquetes. Com a pirólise, os briquetes adquirem maiores teores de carbono e poder calorífico, podendo ser utilizados com maior eficiência na geração de calor e potência (Luengo e Bezzon, 1997).

O conhecimento sobre a cinética de pirólise é essencial para prever o comportamento de processo e na concepção do reator adequado (Damartzis e Zabaniotou, 2011). Do ponto de vista teórico, o objetivo da análise cinética dos processos estimados termicamente é a interpretação do tripleto cinético derminado experimentalmente: a energia de ativação, a frequência de vibração do complexo ativado (o fator pré-exponencial) e o grau de conversão. Já do ponto de vista computacional, a finalidade é estabelecer relações matemáticas entre o grau de conversão, a temperatura e a velocidade de processamento (Vyazovkin et al., 2011). Para isso, muitos modelos têm sido propostos para determinar os parâmetros cinéticos na fase de devolatilização, apresentando um excelente desempenho no intervalo de temperatura de $200-450^{\circ} \mathrm{C}$ ou na faixa de conversão entre 0,15 e 0,80 (Chen et al., 2013).

Diante do exposto, o objetivo deste trabalho é contribuir com o estudo cinético da reação de pirólise do bagaço de laranja, utilizando para isso, análises termogravimétricas para estimar os parâmetros cinéticos a partir dos modelos isoconversionais de Ozawa, Kissinger-Akahira-Sunose e Starink.

\section{METODOLOGIA}

\subsection{Análise termogravimétrica}

Inicialmente, o bagaço de laranja foi cortado em pedaços e colocada em uma estufa por 24 horas a $105^{\circ} \mathrm{C}$, sendo então, macerado em um moinho de alta energia passando para uma granulometria em pó. O material foi submetido a um TGA-50 (Shimadzu Thermogravimetric Analyzer), fluxo de gás nitrogênio $40 \mathrm{ml} / \mathrm{min}$ e massa inicial de aproximadamente $15 \mathrm{mg}$, com taxas de $5,10,20,30 \mathrm{~K} / \mathrm{min}$ de $25^{\circ} \mathrm{C}$ (temperatura ambiente) até a temperatura máxima de $900^{\circ} \mathrm{C}$.

\subsection{Modelos isoconversionais}

O modelo usado na determinação da cinética do fenômeno de decomposição térmica de um sólido é baseado em uma equação que representa a taxa de conversão e pode ser escrita em função de $\mathrm{k}(\mathrm{T})$ e $\mathrm{f}(\mathrm{X})$, como mostrado abaixo (Vyazovkin et al., 2011): 
$\frac{d X}{d t}=k(T) f(X)$

Em que $f(X)$ é o termo dependente da conversão e $X$ representa fração de massa total perdida no processo, também chamada de devolatilização e pode ser definida como:

$X=\frac{m_{0}-m}{m_{0}-m_{\infty}}$

Em que, $\mathrm{m}_{0}$ é a massa inicial de sólido, $\mathrm{m}$ é a massa de sólido em um dado instante de tempo e $\mathrm{m}_{\infty}$ é a massa residual de sólido. $\mathrm{k}(\mathrm{T})$ é a da taxa de reação e sua dependência em relação à temperatura é parametrizada pela equação de Arrhenius:

$k(T)=k_{0} e^{-\frac{E_{a}}{R T}}$

Sendo, $\mathrm{E}_{\mathrm{a}}$ é a energia de ativação, $\mathrm{k}_{0}$ é o fator pré-exponencial, $\mathrm{R}$ é a constante universal dos gases e $\mathrm{T}$ é a temperatura da amostra. Considerando que a degradação térmica do material sólido inicia a temperatura $T_{0}$ e é conduzida por termogravimetria com aumento linear de temperatura $\left(\mathrm{T}=\mathrm{T}_{0}+\beta \mathrm{t}\right)$, então a taxa de aquecimento da amostra, $\beta$, pode ser definida como:

$\beta=\frac{d T}{d t}$

Substituindo-se as equações 3 e 4 na equação 1, e integrando-se as parcelas nos limites de $\propto_{0}-\propto_{\text {máx }}$ e $T_{0}-T_{\text {máx }}$, sendo $T_{o}$ a temperatura inicial (correspondente $a \propto_{0}=0$ ) e $T_{\text {máx }}$ a temperatura de inflexão (correspondente a $\propto_{\text {máx }}$ ), obtém-se a variação do grau de transformação de um sólido em função da temperatura:

$g(X)=\int_{0}^{X} \frac{d X}{f(X)}=\frac{k_{0}}{\beta} \int_{T_{0}}^{T} e^{-\frac{E}{R T}} d T$

Devido a complexidade da reação de pirólise, a forma da função $f(X)$ pode ser simplificada admitindo que a reação segue uma cinética de primeira ordem:

$f(X)=(1-X)$

Os métodos isoconversionais propostos para este tratalho, Ozawa (Ozawa, 1965), Starink (Starink, 1996) e Kissinger-Akahira-Sunose (Akahira e Sunose, 1971), seguem as mesmas considerações físico-químicas e matemáticas, diferindo apenas quanto ao método de aproximação da integração da Equação 5. A equações geradas pela integração da Equação 5, para cada método, são mostradas na Tabela 1.

Tabela 1 - Equações correspondentes aos diferentes métodos isoconversionais.

\begin{tabular}{cc}
\hline Método & Equação \\
\hline Ozawa & $\ln (\beta)=-1,0518\left(E_{a} / R T\right)+\left[\ln \left(k_{0} E_{a} / R\right)-\ln g(x)-5,3305\right]$ \\
Starink & $\ln \left(\beta / T_{x}^{1,8}\right)=-\left(1,007-1,210^{-5} E_{a}\right)\left(E_{a} / R T\right)+c_{2}$ \\
K-A-S & $\ln \left(\beta / T_{x}^{2}\right)=-\left(E_{a} / R T\right)+\ln \left[k_{0} R /\left(E_{a} g(x)\right)\right]$ \\
\hline
\end{tabular}




\section{RESULTADOS E DISCUSSÃO}

\subsection{Análise termogravimétrica dinâmica}

As curvas referentes à perda de massa (TG) e a taxa da perda de massa (DTG) nos ensaios dinâmicos para a amostra de bagaço de laranja, nas taxas de aquecimento de 5, 10, 20 e $30 \mathrm{~K} / \mathrm{min}$, são mostradas na Figura 1.

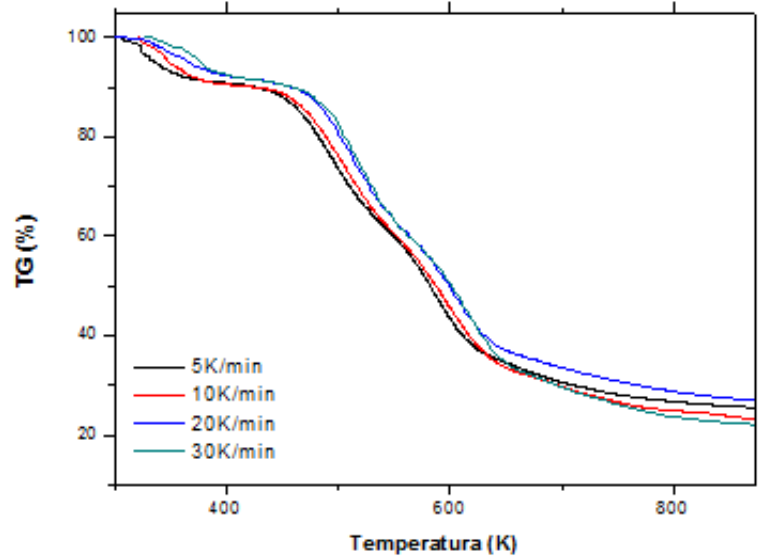

(a)

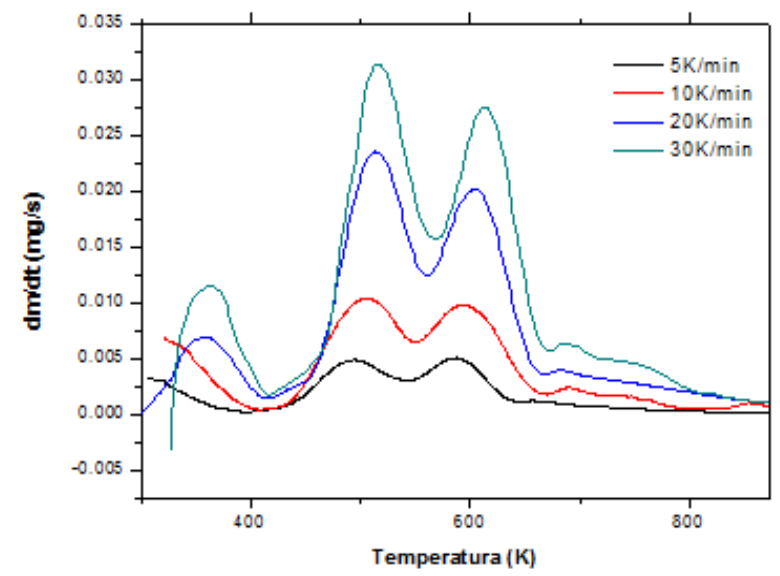

(b)

Figura 1 - Curvas de (a) TG e (b) DTG para o bagaço de laranja.

Na Figura 1(a) é possível observar que houve duas fases no processo de pirólise do bagaço de laranja. A primeira refere-se à perda de massa devido à perda de umidade do material até aproximadamente 393,15K. A segunda fase faz referência à devolatilização da amostra, inicialmente com um lento processo de perda de massa $(393,15-423,15 \mathrm{~K})$, após, a curva de TG caiu drasticamente, entre 423,15 e 673,15K, devido à ocorrência de reações químicas complexas e, por fim, a partir de $673,15 \mathrm{~K}$ os resíduos foram lentamente decompostos. A partir da Figura 1(b) observa-se também a decomposição de hemicelulose no intervalo de temperatura de aproximadamente, $423,15-580 \mathrm{~K}$, a de celulose entre $580-673,15 \mathrm{~K}$ e por fim, a de lignina, embora, a mesma se decomponha em toda faixa de temperatura do processo de devolatilização.

\subsection{Métodos isoconversionais - Cálculo da energia de ativação global}

As Figuras 2, a, b e c, mostram as regressões lineares referentes respectivamente, aos métodos de Ozawa, Starink e Kissinger-Akahira-Sunose (K-A-S), para a reação de pirólise do bagaço de laranja na faixa de conversão de 0 a $80 \%$ com intervalo de $5 \%$.

Segundo Chen et al. (2013), nos mesmos níveis de conversão, os gráficos de Arrhenius para os modelos cinéticos, no caso do método de Ozawa por exemplo, os gráficos de $\ln (\beta)$ versus $1 / \mathrm{T}$ devem ter um comportamento linear, a partir do qual a energia de ativação pode ser calculada. De forma análoga, o mesmo procedimento foi realizado para os métodos de Starink e K-A-S. Ainda, em geral, de acordo com os autores, três ou mais taxas de aquecimento são utilizadas para a obtenção de uma série de valores de energia de ativação. 


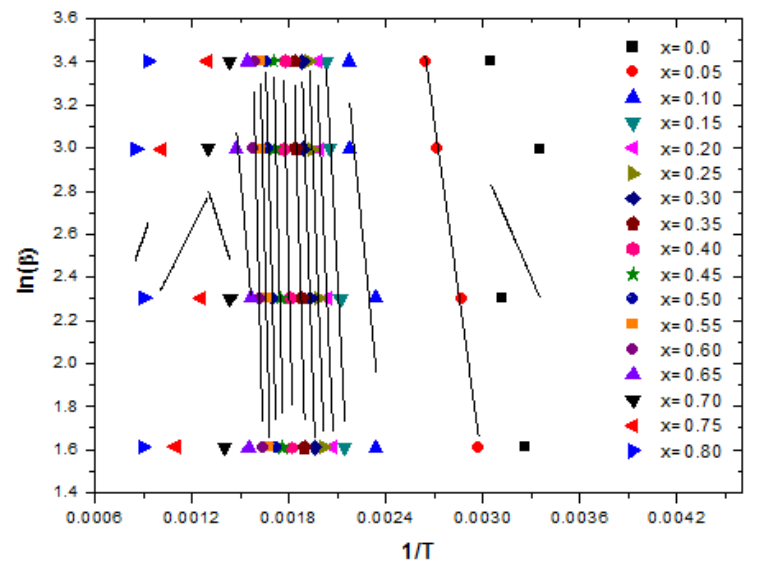

(a)

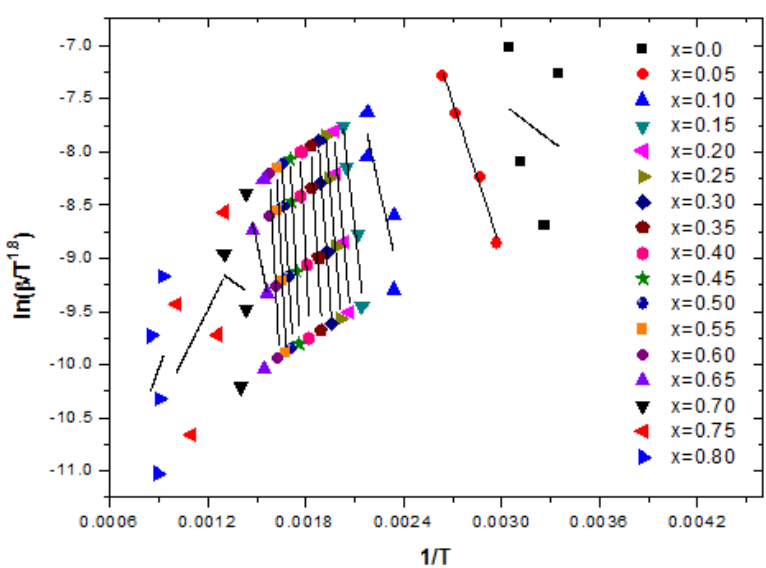

(b)

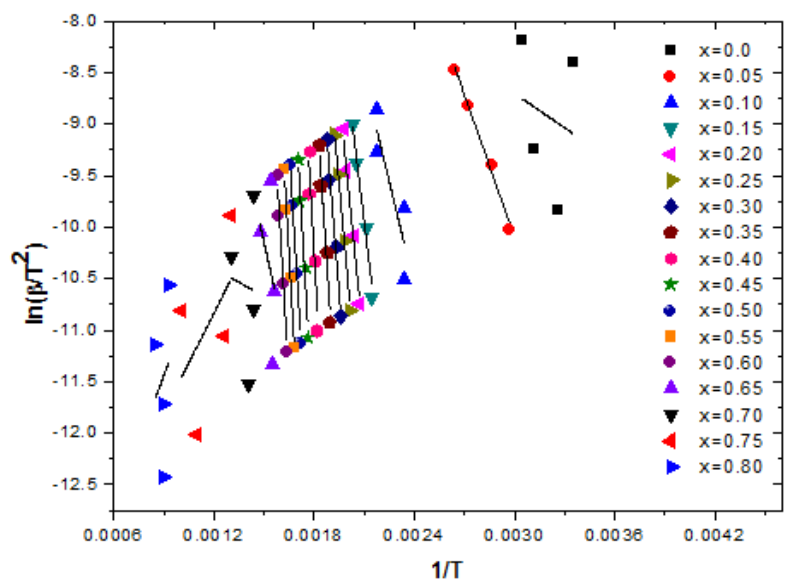

(c)

Figura 2-Gráficos de Arrhenius para os métodos de: (a) Ozawa, (b) Starink e (c) K-A-S, para o bagaço de laranja em diferentes conversões.

Em todos os métodos apresentados na Figura 2 foram observadas boas correlações para as diferentes conversões de 15 a $60 \%$, o paralelismo denota que o processo de decomposição térmica foi bem descrito por esses modelos, indicando que provavelmente o mecanismo de reação para essa faixa de conversão é o mesmo ou há uma unificação dos mecanismos de reações múltiplas. Entretanto, o não paralelismo nas faixas de 0 a $10 \%$ e de 65 a $80 \%$, para todos os métodos, indica que existem mudanças nos mecanismos de reação, acarretando em diferentes valores para a energia de ativação.

Nas conversões entre 0 e $10 \%$, o mecanismo de reação consiste na perda de água da biomassa. Segundo Chen et al. (2013), este é um processo físico e não químico fazendo com que os modelos cinéticos de pirólise baseados nas reações químicas não sejam adequados para a fase de secagem e assim sendo, os valores da energia de ativação obtidos para esta faixa foram retirados dos cálculos.

$\mathrm{Na}$ Tabela 2 estão apresentadas as energias de ativação calculadas para cada valor de conversão utilizando-se os métodos de K-A-S, Starink e Ozawa para a reação de pirólise do 
bagaço da laranja. Os valores dos coeficientes de determinação $\left(\mathrm{r}^{2}\right)$ mostrados na Tabela 2, para todos os modelos, são adequados para as estimativas de energia de ativação em uma faixa de conversão de $15-60 \%\left(0,84 \leq \mathrm{r}^{2} \leq 0,95\right)$, com exc eção dos valores obtidos para as conversões de 40 e $60 \%$. Os valores dos ajustes lineares, para os intervalos de conversão de 0 a $10 \%$ e de 65 a $80 \%$, de forma geral, foram baixos atingindo valores mínimos de 0,01 .

Tabela 2 - Energias de ativação para cada valor de conversão, calculadas pelos métodos de Ozawa, Starink, e K-A-S, para o bagaço de laranja.

\begin{tabular}{|c|c|c|c|c|c|c|}
\hline \multirow{2}{*}{$\mathbf{x}$} & \multicolumn{2}{|l|}{ Ozawa } & \multicolumn{2}{|c|}{ Starink } & \multicolumn{2}{|c|}{ K.A.S } \\
\hline & Ea $\left(\right.$ KJ.mol $\left.{ }^{-1}\right)$ & $\mathbf{r}^{2}$ & Ea $\left(\right.$ KJ.mol $\left.{ }^{-1}\right)$ & $\mathbf{r}^{2}$ & Ea (KJ.mol $\left.{ }^{-1}\right)$ & $\mathbf{r}^{2}$ \\
\hline 0,0 & 13,72 & 0,13 & 9,69 & 0,19 & 9,23 & 0,19 \\
\hline 0,05 & 42,05 & 0,98 & 38,67 & 0,98 & 38,30 & 0,98 \\
\hline 0,10 & 60,13 & 0,54 & 56,28 & 0,47 & 55,87 & 0,46 \\
\hline 0,15 & 113,66 & 0,91 & 111,73 & 0,90 & 111,58 & 0,90 \\
\hline 0,20 & 133,81 & 0,91 & 132,56 & 0,90 & 132,51 & 0,90 \\
\hline 0,25 & 150,50 & 0,95 & 149,82 & 0,94 & 149,85 & 0,94 \\
\hline 0,30 & 169,61 & 0,94 & 169,61 & 0,93 & 169,72 & 0,93 \\
\hline 0,35 & 201,76 & 0,86 & 203,00 & 0,85 & 203,26 & 0,85 \\
\hline 0,40 & 234,14 & 0,61 & 236,55 & 0,59 & 236,99 & 0,59 \\
\hline 0,45 & 229,06 & 0,85 & 230,95 & 0,84 & 231,33 & 0,84 \\
\hline 0,50 & 216,85 & 0,90 & 217,94 & 0,89 & 218,22 & 0,89 \\
\hline 0,55 & 236,24 & 0,93 & 238,02 & 0,93 & 238,40 & 0,93 \\
\hline 0,60 & 228,17 & 0,77 & 229,35 & 0,75 & 229,64 & 0,75 \\
\hline 0,65 & 67,61 & 0,05 & 60,88 & 0,08 & 60,13 & 0,09 \\
\hline 0,70 & 18,95 & 0,20 & 8,91 & 0,24 & 7,74 & 0,24 \\
\hline 0,75 & $-11,71$ & 0,16 & $-25,19$ & 0,02 & $-26,78$ & 0,01 \\
\hline 0,80 & $-17,66$ & 0,24 & $-35,25$ & 0,21 & $-37,33$ & 0,20 \\
\hline
\end{tabular}

Nas Figuras 3, a, b e c, estão apresentadas a distribuição de energia de ativação para todo o processo de pirólise do bagaço de laranja, respectivamente, para os métodos de Ozawa, Starink e K.A.S.

Os valores de energia de ativação obtidos nas faixas de conversão de 0 a $10 \%$ e de 65 a $80 \%$ foram retirados para o cálculo da energia de ativação global, já que foi observada pouca ou nenhuma correlação e, de forma geral, foram obtidos baixos valores de $r^{2}$. Ainda, com o auxílio da Figura 3, observa-se que as faixas de conversão extremas apresentam valores de energia de ativação bem inferiores se comparados aos valores na faixa de 15 a $60 \%$, para todos os métodos. Assim, as médias de energia de ativação obtidas com os dados experimentais para o bagaço de laranja foram 191,38, 191,96 e 192,15 KJ.mol-1 para os métodos de Ozawa, Starink e K-A-S, respectivamente. 


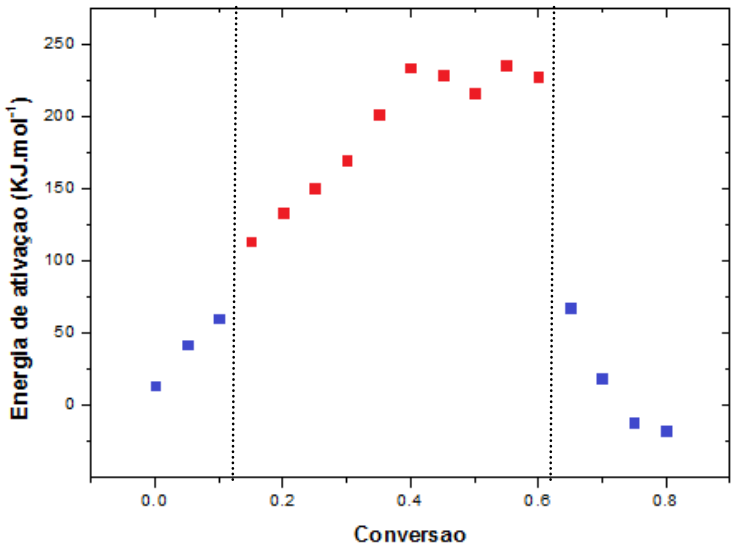

(a)

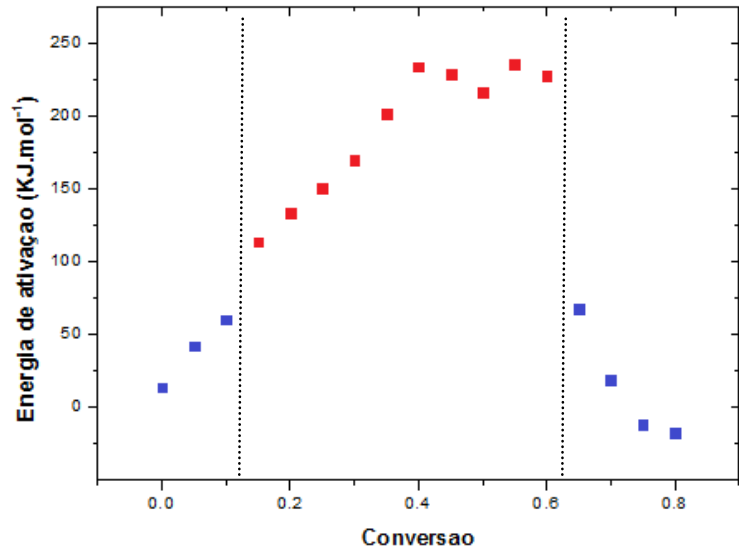

(b)

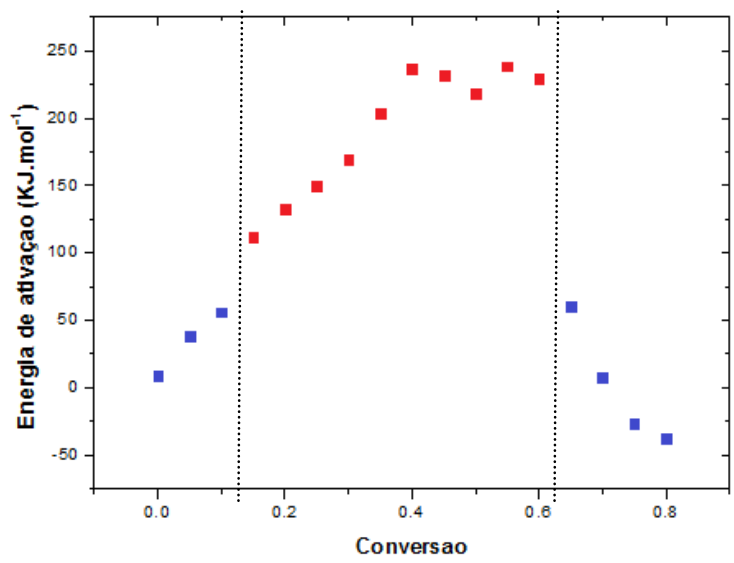

(c)

Figura 3 - Distribuição da energia de ativação com diferentes conversões para os métodos de (a) Ozawa, (b) Starink e (c) K.A.S.

\section{CONCLUSÃO}

A partir da curva DTG foi possível observar duas fases na pirólise do bagaço de laranja. A primeira ocorre devido à perda de água do bagaço. Já a segunda refere-se a degradação de hemicelulose e celulose, neste sequência, e de liginina por todo processo. Os valores de energia de ativação glogal média obtidos para o processo de pirólise pelos métodos isoconversionais, Ozawa, Starink e K.A.S foram próximos e adequados, visto que, os valores dos coeficientes de determinação foram satisfatórios no intervalo de conversão de 15 a $60 \%$.

\section{REFERÊNCIAS}

AKAHIRA, T.; SUNOSE, T. Joint convention of four electrical institutes. Research Report Chiba Institute and Technology, v. 16, p. 22-31, 1971.

ANEEL - Agencia Nacional de Energia Elétrica. Atlas de Energia Elétrica - 2a ed., Brasília: 2005. Disponível em: <http://www.aneel.gov.br/aplicacoes/Atlas/download.htm>. Acesso em: 05 abr. 2014. 
CHEN, D, ZHENG, Y, ZHU, X. In-depth investigation on the pyrolysis kinetics of raw biomass, Part I: Kinetic analysis for the drying and devolatilization stages, Bioresource Technology, v. 131, p. 40-46, China, 2013.

CITRUSBR - ASSOCIAÇÃO NACIONAL DE EXPORTADORES DE SUCOS CÍTRICOS. O retrato da citricultura brasileira. Disponível em: <http://www.citrusbr.com.br/exportadorescitricos/saiba-mais/o-retrato-da-citricultura-brasileira-189513-1.asp>. Acesso em: 14 abr. 2014.

CORAZZA, M.; RODRIGUES, D.G.; NOZAKI, J. Quim. Nova, v. 24, p. 449-452, 2001.

Damartzis, T., Zabaniotou, A. Thermochemical conversion of biomass to second generation biofuels through integrated process design - A review, Renewable Sustainable Energy, v. 15, p. 366-378, 2011.

GAMBA, M. Produção de Biodiesel através de catálise enzimática em líquido iônico. 2009. Dissertação (Mestrado em Química) - Programa de Pós-Graduação em Química, Universidade Federal do Rio Grande do Sul, Porto Alegre, 2009.

IBGE - INSTITUTO BRASILEIRO DE GEOGRAFIA E ESTATÍSTICAS. Sistema de Recuperação Automática - $\quad$ SIDRA. Disponível em: $<$ http://www.sidra.ibge.gov.br/bda/tabela/listabl.asp?c=1613\&z=t\&o=11>. Acesso em: 10 abr. 2014.

LUENGO, C. A.; BEZZON, G. Energéticos derivados da biomassa. In: CORTEZ, L. A. B.; LORA, E. S. (Coord.). Tecnologias de conversão energética da biomassa. Manaus: EDUA, EFEI, 1997.

OZAW A, T. A new method of analyzing thermogravimetric data. Bull. Chem. Soc. Japan, v. 38, p. $1881,1965$.

SILVEIRA, M. S. Aproveitamento das cascas de coco verde para produção de briquete em salvador - BA. 2008. Dissertação (Mestrado em Gerenciamento e Tecnologias Ambientais no Processo Produtivo) - Programa de Pós-Graduação em Gerenciamento e Tecnologias Ambientais no Processo Produtivo - Ênfase em Produção Limpa, Universidade Federal da Bahia, Salvador, 2008 .

STARINK, M. J. A New Method for the Derivation of Activation-Energies from Experiments Performed at Constant Heating Rate, Thermochim. Acta, v. 288, p. 97, 1996.

VYAZOVKIN, S, et al. ICTAC Kinetics Committee recommendations for performing kinetic computations on thermal analysis data, Thermochimica Acta, v. 520, p. 1-19, 2011. 